Meeting Report

\title{
Meeting Report: Aging Research and Drug Discovery
}

Esther Meron ${ }^{1,}{ }^{*}$, Maria Thaysen ${ }^{1,}{ }^{*}$, Suzanne Angeli², Adam Antebi ${ }^{3}$, Nir Barzilai ${ }^{4,5}$, Joseph A. Baur $^{6}$, Simon Bekker-Jensen ${ }^{1}$, Maria Birkisdottir ${ }^{7,8}$, Evelyne Bischof ${ }^{9}$, Jens Bruening ${ }^{10}$, Anne Brunet $^{11}$, Abigail Buchwalter ${ }^{12}$, Filipe Cabreiro ${ }^{13,14}$, Shiqing Cai ${ }^{15}$, Brian H. Chen ${ }^{16,17}$, Maria Ermolaeva $^{18}$, Collin Y. Ewald ${ }^{19}$, Luigi Ferrucci ${ }^{20}$, Maria Carolina Florian ${ }^{21}$, Kristen Fortney ${ }^{22}$, Adam Freund $^{23}$, Anastasia Georgievskaya ${ }^{24}$, Vadim N. Gladyshev ${ }^{25}$, David Glass ${ }^{26}$, Tyler Golato ${ }^{27}$, Vera Gorbunova $^{28}$, Jan Hoejimakers ${ }^{29}$, Riekelt H. Houtkooper ${ }^{30}$, Sibylle Jager ${ }^{31}$, Frank Jaksch ${ }^{32}$, Georges Janssens ${ }^{30}$, Martin Borch Jensen ${ }^{33}$, Matt Kaeberlein ${ }^{34}$, Gerard Karsenty ${ }^{35}$, Peter de Keizer $^{36}$, Brian Kennedy,37,38, James L. Kirkland ${ }^{39}$, Michael Kjaer ${ }^{40}$, Guido Kroemer ${ }^{41}$, Kai-Fu Lee ${ }^{42}$, Jean-Marc Lemaitre ${ }^{43}$, David Liaskos ${ }^{44}$, Valter D. Longo ${ }^{45}$, Yu-Xuan Lư ${ }^{3}$, Michael R. MacArthur ${ }^{46}$, Andrea B. Maier ${ }^{38,47,48}$, Christina Manakanatas ${ }^{49}$, Sarah J. Mitchell ${ }^{46}$, Alexey Moskalev ${ }^{50,51}$, Laura Niedernhofer ${ }^{52}$, Ivan Ozerov ${ }^{53}$, Linda Partridge ${ }^{3}$, Emmanuelle Passegué ${ }^{54}$, Michael A. Petr ${ }^{1,55}$, James Peyer ${ }^{56}$, Dina Radenkovic ${ }^{57}$, Thomas A. Rando ${ }^{58}$, Suresh Rattan ${ }^{59}$, Christian G. Riedel ${ }^{60}$, Lenhard Rudolph ${ }^{18}$, Ruixue Ai ${ }^{61}$, Manuel Serrano ${ }^{62}$, Björn Schumacher ${ }^{14}$, David A. Sinclair ${ }^{63}$, Ryan Smith $^{64}$, Yousin Suh ${ }^{65}$, Pam Taub ${ }^{66}$, Alexandre Trapp ${ }^{25}$, Anne-Ulrike Trendelenburg ${ }^{67}$, Dario Riccardo Valenzano ${ }^{3,18}$, Kris Verburgh ${ }^{68}$, Eric Verdin², Jan Vijg ${ }^{4}$, Rudi G.J. Westendorp ${ }^{69}$, Alessandra Zonari $^{70}$, Daniela Bakula ${ }^{1}$, Alex Zhavoronkov ${ }^{53}$, Morten Scheibye-Knudsen ${ }^{1}$

${ }^{1}$ Center for Healthy Aging, Department of Cellular and Molecular Medicine, University of Copenhagen, Copenhagen, Denmark

${ }^{2}$ Buck Institute for Research on Aging, Novato, CA 94945, USA

${ }^{3}$ Max Planck Institute for Biology of Ageing, Cologne, Germany

${ }^{4}$ Department of Genetics, Albert Einstein College of Medicine, Bronx, NY 10461, USA

${ }^{5}$ Institute for Aging Research, Department of Medicine, Albert Einstein College of Medicine, Bronx, NY 10461, USA

${ }^{6}$ Smilow Center for Translational Research, University of Pennsylvania, Philadelphia, PA 19104, USA

${ }^{7}$ Department of Molecular Genetics, Erasmus MC, Rotterdam, Netherlands

${ }^{8}$ Department of Neuroscience, Erasmus MC, Rotterdam, Netherlands

${ }^{9}$ Shanghai University of Medicine and Health Sciences, College of Clinical Medicine, Shanghai, China

${ }^{10}$ Max Planck Institute for Metabolism Research, Cologne, Germany

${ }^{11}$ Department of Genetics, Stanford School of Medicine, Stanford University, Stanford, CA 94305, USA

${ }^{12}$ Cardiovascular Research Institute, University of California, San Francisco, CA 94158, USA

${ }^{13}$ Institute of Clinical Sciences, Imperial College London, Hammersmith Hospital Campus, London W12 ONN, UK

${ }^{14}$ CECAD Research Center, Faculty of Medicine, University of Cologne, Cologne, Germany

${ }^{15}$ Institute of Neuroscience, Chinese Academy of Science, Shanghai, China

${ }^{16}$ FOXO Technologies Inc, Minneapolis, MN 55402, USA

${ }^{17}$ The Herbert Wertheim School of Public Health and Human Longevity Science, UC San Diego, La Jolla, CA 92093, USA

${ }^{18}$ Leibniz Institute on Aging, Jena, Germany

${ }^{19}$ Laboratory of Extracellular Matrix Regeneration, Institute of Translational Medicine, Department of Health Sciences and Technology, ETH Zürich, Schwerzenbach CH-8603, Switzerland

${ }^{20}$ Longitudinal Studies Section, Translational Gerontology Branch, National Institute on Aging, National Institutes of Health, Baltimore, MD 21224, USA

${ }^{21}$ Program for Regenerative Medicine, IDIBELL, Barcelona, Spain

${ }^{22}$ BioAge Labs, Inc., Richmond, CA 94804, USA

${ }^{23}$ Arda Therapeutics, San Francisco Bay Area, San Carlos, CA 94070, USA

${ }^{24}$ HautAI OÜ, Tallinn, Estonia 
${ }^{25}$ Division of Genetics, Department of Medicine, Brigham and Women's Hospital, Harvard Medical School, Boston, MA 02115, USA

${ }^{26}$ Regeneron Pharmaceuticals, Inc., Tarrytown, NY 10591, USA

${ }^{27}$ Molecule, Schaffhausen, Switzerland

${ }^{28}$ Departments of Biology and Medicine, University of Rochester, Rochester, NY 14627, USA

${ }^{29}$ Department of Genetics, Erasmus MC, University Medical Center Rotterdam, Rotterdam, The Netherlands

${ }^{30}$ Laboratory Genetic Metabolic Diseases, Amsterdam UMC, University of Amsterdam, Amsterdam, The Netherlands

${ }^{31}{ }^{\prime}$ 'Oréal Research and Innovation, Aulnay-sous-Bois, France

${ }^{32}$ ChromaDex Corp, Los Angeles, CA 90024, USA

${ }^{33}$ Gordian Biotechnology, San Francisco, CA 94107, USA

${ }^{34}$ Departments of Laboratory Medicine and Pathology, University of Washington, Seattle, WA 98195, USA

${ }^{35}$ Department of Genetics and Development, Columbia University Medical Center, New York, NY 10032, USA

${ }^{36}$ Department of Molecular Cancer Research, Center for Molecular Medicine, Division of Biomedical Genetics, University Medical Center Utrecht, Utrecht University, Utrecht, The Netherlands

${ }^{37}$ Departments of Biochemistry and Physiology, Yong Loo Lin School of Medicine, National University Singapore,

Singapore

${ }^{38}$ Center for Healthy Longevity, National University Health System, Singapore

${ }^{39}$ Division of General Internal Medicine, Department of Internal Medicine, Mayo Clinic, Rochester, MN 55905, USA

${ }^{40}$ Department of Clinical Medicine, University of Copenhagen, Copenhagen, Denmark

${ }^{41}$ Centre de Recherche des Cordeliers, Université de Paris, Sorbonne Université, Inserm U1138, Paris, France

${ }^{42}$ Sinovation Ventures and Sinovation Al Institute, Beijing, China

${ }^{43}$ Institute for Regenerative Medicine and Biotherapies, INSERM UMR 1183, Montpellier, France

${ }^{44} \mathrm{Nagi}$ Bioscience, Ecublens, Switzerland

${ }^{45}$ USC Davis School of Gerontology, University of Southern California, Los Angeles, CA 90089, USA

${ }^{46}$ Department of Health Sciences and Technology, ETH Zurich, Zurich, Switzerland

${ }^{47}$ Department of Human Movement Sciences, @AgeAmsterdam, Faculty of Behavioural and Movement Sciences, Amsterdam Movement Sciences, Vrije Universiteit Amsterdam, Amsterdam, The Netherlands

${ }^{48}$ Department of Medicine, Yong Loo Lin School of Medicine, National University Singapore, Singapore

${ }^{49}$ Max Perutz Labs, The University of Vienna, Vienna, Austria

${ }^{50}$ Institute of Biology of FRC Komi Science Center of Ural Division of RAS, Syktyvkar, Russia

${ }^{51}$ Russian Clinical and Research Center of Gerontology, Moscow, Russia

${ }^{52}$ Institute on the Biology of Aging and Metabolism, Department of Biochemistry, Molecular Biology and

Biophysics, University of Minnesota, Minneapolis, MN 55455, USA

${ }^{53}$ Insilico Medicine, Hong Kong Science and Technology Park, Hong Kong

${ }^{54}$ Columbia Stem Cell Initiative, Columbia University, New York, NY 10032, USA

${ }^{55}$ Tracked.bio, Copenhagen, Denmark

${ }^{56}$ Cambrian Biopharma, New York, NY 11205, USA

${ }^{57}$ Hooke London by Health and Longevity Optimisation, London, UK

${ }^{58}$ Department of Neurology and Neurological Sciences and Paul F. Glenn Center for Biology of Aging, Stanford University School of Medicine, Stanford, CA 94305, USA

${ }^{59}$ Department of Molecular Biology and Genetics, Aarhus University, Aarhus, Denmark

${ }^{60}$ Department of Biosciences and Nutrition, Karolinska Institute, Stockholm, Sweden

${ }^{61}$ Department of Clinical Molecular Biology | UiO, University of Oslo and Akershus University Hospital, Norway

${ }^{62}$ Institute for Research in Biomedicine (IRB Barcelona), Barcelona Institute of Science and Technology (BIST),

Catalan Institution for Research and Advanced Studies (ICREA), Barcelona, Spain

${ }^{63}$ Blavatnik Institute, Department of Genetics, Paul F. Glenn Center for Biology of Aging Research at Harvard

Medical School, Boston, MA 94107, USA

${ }^{64}$ TruDiagnostic, Lexington, KY 40503, USA

${ }^{65}$ Departments of Obstetrics and Gynecology, Genetics and Development, Columbia University, New York, NY 10027, USA 


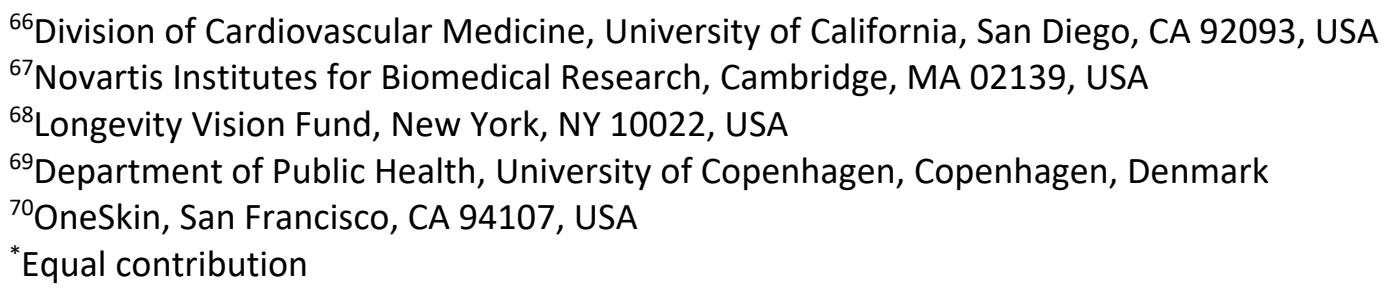

Correspondence to: Daniela Bakula, Alex Zhavoronkov, Morten Scheibye-Knudsen; email: bakula@sund.ku.dk, alex@insilico.com, mscheibye@sund.ku.dk

Keywords: aging, drug discovery, conference, Al, longevity

Received: January 4, $2022 \quad$ Accepted: January 11, 2022

Published: January 28, 2022

Copyright: (C) 2022 Meron et al. This is an open access article distributed under the terms of the Creative Commons Attribution License (CC BY 3.0), which permits unrestricted use, distribution, and reproduction in any medium, provided the original author and source are credited.

\begin{abstract}
Aging is the single largest risk factor for most chronic diseases, and thus possesses large socioeconomic interest to continuously aging societies. Consequently, the field of aging research is expanding alongside a growing focus from the industry and investors in aging research. This year's 8th Annual Aging Research and Drug Discovery (ARDD) meeting was organized as a hybrid meeting from August 30th to September 3rd 2021 with more than 130 attendees participating on-site at the Ceremonial Hall at University of Copenhagen, Denmark, and 1800 engaging online. The conference comprised of presentations from 75 speakers focusing on new research in topics including mechanisms of aging and how these can be modulated as well as the use of Al and new standards of practices within aging research. This year, a longevity workshop was included to build stronger connections with the clinical community.
\end{abstract}

\section{INTRODUCTION}

As the world population ages, the need for a deeper understanding of healthy aging is becoming increasingly important. In recent years, the Aging Research and Drug Discovery (ARDD) meeting has brought experts of different sectors together, thereby providing researchers and representatives from the industry a platform to exchange ideas, thoughts and expertise and to foster collaborations. This year the 8th ARDD conference, organized by Morten Scheibye-Knudsen, University of Copenhagen, Alex Zhavoronkov, Insilico Medicine and Daniela Bakula, University of Copenhagen, was held in Copenhagen, Denmark from August 30th to September 3rd 2021. The meeting featured presentations from 75 world leading aging experts with participation of over 2000 attendees, attending either in person or virtually, as well as a Longevity Medicine Workshop and a panel session of venture capitalists. As a new initiative, the Inspire Longevity program was started this year, aiming to inspire young students to engage in longevity research.

This report covers the many exciting research areas presented at the conference, ranging from cellular maintenance pathways and how they can be modulated to promote healthy aging, new thoughts on stem cell rejuvenation and senotherapeutics, to new model systems in aging. It explores the many advances in AI and aging clocks, and the innovations in drug discovery from preclinical to clinical studies.

\section{Age-dependent control of cellular maintenance processes}

Understanding cellular maintenance processes such as the regulation of genome stability and proteostasis is an important area of aging research [1]. With age there is an accumulation of somatic mutations known as somatic mosaicism, creating a mutational burden that can lead to decreased transcriptional stability. Jan Vijg, Albert Einstein College of Medicine, USA, presented new advances in single cell sequencing and multi-omics that allows the study of de novo mutations leading to somatic mosaicism [2]. Björn Schumacher, University of Cologne, Germany, highlighted DNA damage as a main driver of aging. Expanding on the crosstalk between germline and somatic cell maintenance, he showed that DNA damage induced $\mathrm{H} 3 \mathrm{~K} 4 \mathrm{me} 2$ in C. elegans promotes recovery of protein biosynthesis and homeostasis thereby linking DNA repair and 
protein homeostasis [3]. Notably, there is also a correlation between protein fidelity and lifespan in different organisms. Filipe Cabreiro, Imperial College London, UK, and University of Cologne, Germany, discovered the ribosomal protein S23 (RPS23) K60R mutation to be a hyperaccuracy mutant. RPS23 K60R mutation leads to improved translation accuracy, heat resistance, and longer lifespan in $S$. pombe, C. elegans and $D$. melanogaster without reducing translation, making translational fidelity a potential therapeutic target [4]. Notably, protein stability varies across tissues with age independently of cell proliferation rate, which suggests shared and unique aging vulnerabilities of proteins across tissues, as presented by Abigail Buchwalter, University of California, USA [5].

\section{Longevity pathways}

Cellular pathways associated with increased longevity have been extensively studied for many years. Consequently, drugs targeting these pathways have been shown to have a positive effect on lifespan in different model organisms.

Rapamycin is known to increase the lifespan of organisms by acting through mTOR. Linda Partridge, Max Planck Institute for Biology of Ageing, Germany, highlighted the importance of intestinal homeostasis and aging. Their research revealed that short term and early treatment with rapamycin extends lifespan in D. melanogaster as much as chronic rapamycin treatment. The short-term rapamycin treatment induced long-term increase in autophagy, decrease in pathology of fly intestine. Similarly, short term rapamycin treatment in mice caused a reduction in intestinal pathology for up to 6 months after rapamycin treatment (unpublished data). Moreover, there is an unconventional intestine sex-specific TORC1-histone axis which uncovers a new aspect to the improved longevity with rapamycin as shown by $\mathrm{Yu}$-Xuan Lu from Max Planck Institute for Biology of Ageing, Germany. The observed sex differences and possible other unconventional pathways need to be considered prior to rapamycin treatment [6].

Another well studied pathway in aging is nicotinamide adenine dinucleotide (NAD) metabolism and the agedependent NAD decline. Joseph A. Baur, University of Pennsylvania, USA, showed that the mitochondrial protein SLC25A51 is a transporter that modulates the transport of NAD into the mitochondria. An AI-based drug screen revealed promising results that this transporter can be modulated and in the future be a novel way of preventing age-dependent NAD decline [7]. Traditionally, the NAD pathway has been modulated by supplementing with NAD precursors such as nicotinamide riboside (NR). Frank Jaksch from
ChromaDex Corp. suggested that NR supplementation could be a potential treatment for orphan diseases characterized by DNA damage and mitochondrial dysfunction which has been demonstrated in preclinical models. Currently, clinical studies are examining the potential effects of NR supplementation [8, 9]. However, Riekelt Houtkooper's group, Amsterdam $\mathrm{UMC}$, Netherlands, point to NAD+ precursors having shown limited translatability in human trials and has instead characterized a novel reduced NAD + precursor, $\mathrm{NMNH}$. NMNH increases NAD+ levels to a greater extent than traditional precursors NR and NMN and sustains elevated NAD+ in mice [10].

Brain aging is associated with behavioral deterioration and may be improved by enhancing levels of neurotransmitters. Shiqing Cai, Institute of Neuroscience, China, utilized a genome-wide RNAi screen in $C$. elegans and found that overexpression of the two epigenetic regulators BAZ2B and EHMT1 may cause decreased levels of serotonin and dopamine and decreased mitochondrial function. Further, the gene expression of both positively correlates with the progression of Alzheimer's disease and are therefore potential novel anti-aging target genes [11]. Another promising anti-aging target is the mitochondrial permeability transition pore ( $\mathrm{mPTP})$, which increases with age and age-related diseases such as Alzheimer's disease, Parkinson's disease, and ischemia/reperfusion injuries. OSCP, a subunit of F-ATP synthase, is an important regulator of the MPTP and loss of OSCP leads to a decreased lifespan in C. elegans and initiation of a maladaptive mitochondrial unfolded protein response $\left(\mathrm{UPR}^{\mathrm{mt}}\right)$. Thus, $\mathrm{mPTP} / \mathrm{UPR}^{\mathrm{mt}}$ may be a contributor of aging and age-related disease as shown by Suzanne Angeli, Buck Institute for Research on Aging, USA [12].

Collin Ewald from ETH Zurich, Switzerland, proposed extracellular matrix homeostasis as a novel longevity pathway that is highly conserved among species. Supplements with components of the extracellular matrix which are well known and safe could therefore be used as a potential longevity pill [13]. Looking at hydrogen sulfide (HSO) homeostasis, Alexey Moskalev, Russian academy of Sciences, Russia, has found that its disruption is associated with aging and therefore a potential gero-therapeutic target. He showed that combined genetic and therapeutic interventions of HSO metabolism led to beneficial effects of HSO production and life- and health span extension in D. melanogaster along with stress resistance [14]. Another novel mechanism to regulate homeostasis at the embryonic level is through adrenal steroidogenesis. Gerard Karsenty, Columbia University, USA, showed that embryonic osteocalcin determines adrenal development. As a result, embryonic but not postnatal 
osteocalcin is essential for life-long maintenance of whole-organism homeostasis through its regulation of blood pressure and electrolyte metabolism. In this capacity embryonic osteocalcin determines healthy aging [15]. Adam Antebi, Max Planck Institute for Biology of Ageing, Germany, presented their latest study in which they used metabolic profiling of longlived C. elegans strains and identified the folate/ methionine cycle as a convergence point of aging pathways. This elucidates how single genes or metabolites can regulate lifespan through multiple pathways [16].

With the high correlation between age and the risk of COVID-19 related deaths, novel research is emerging in aging pathways. Activin signalling has been seen to increase with age, and is especially elevated in acute respiratory disease syndrome (ARDS) patients and with relevance to COVID-19. David Glass, Regeneron, USA showed that Activin A production can be stimulated by a cytokine storm and is seen in most severe COVID-19 patients. Activin A may therefore be a biomarker of increased risk of ARDS in COVID-19 patients. However, Activin A is also associated with decreased viral load in cell culture, and is therefore a problematic therapeutic target [17].

\section{Cellular stress and aging}

Looking at aging as the progressive loss of health treatment should be focused on drugs for health and not against disease, stated Suresh Rattan, Aarhus University, Denmark. In this context, he highlighted the biological response hormesis and how stress induction can upregulate adaptive responses and how hormetins in various forms promote general health [18]. Michael Kjær, University of Copenhagen, Denmark, elaborated on a physical hormetin, exercise. A study of his group revealed that life-long endurance training causes an increased muscle mass maintenance and strength in older individuals. The improved muscle function is possibly evoked by an increased muscle growth and anti-inflammatory effects of physical training. Notably, these muscle improvements can be achieved even when the training is started in old age, though the extent of improvement diminishes with age [19]. During different stress conditions such as endurance training, the cells adapt through different signalling pathways. One signaling pathway is the MAP kinase signalling cascade, and here Simon Bekker-Jensen, University of Copenhagen, Denmark, showed that the two MAP3K isoforms ZAKa and $\mathrm{ZAKb}$ respond to stress and promote adaptive mechanisms. Lack of ZAKa leads to a shorter lifespan in $C$. elegans in response to amino acid starvation. $\mathrm{ZAKb}$ responds to mechanical stress and its deletion in humans is associated with severely impaired muscle function [20] (unpublished).

\section{The benefits of dietary restriction}

Another well-described stressor that has been shown to extend lifespan as well as improve healthspan is dietary restriction (DR). With age there is a decrease in RNA but increase in RNA polymerase II (RNAPII), specifically elongation RNAPII, suggesting lower RNA productivity of RNAPII with age as presented by Jan Hoeijmakers, Erasmus Medical Center Rotterdam, Netherlands. This preferentially affects long genes being suppressed in expression leading to an imbalanced RNA pool. DR compensates for this effect by reducing DNA damage load and alleviating transcription stress (unpublished). Maria Birkisdottir, Erasmus MC, Netherlands, showed that DR but not rapamycin leads to an increased health- and lifespan in $\mathrm{ERCC1}^{-/ \Delta}$ mice. Similarly, DR but not rapamycin prevents the observed degeneration of Purkinje neurons in $\mathrm{ERCC1}^{-/ \Delta}$ mice, indicating that DR and rapamycin differ in their mode of action [21]. Moreover, fasting or a ketogenic diet have been shown by Thomas A. Rando Stanford, USA to cause the muscle stem cells to enter a deep quiescent state mediated through HDAC1 and p53. This causes an increased resilience of aged muscle stem cells [22].

The beneficial effects of DR are lost in late-life interventions, potentially because the induction of mitochondrial function is necessary for the response but declines with age. Lenhard Rudolph, Leibniz Institute on Aging, Germany, showed new insides into new mechanisms that can rescue the capacity of late life DR (administered in old animals) to induce health promoting stress signals and improvements in stem cell function and lifespan (unpublished). Additionally, Maria Ermolaeva, Leibniz Institute on Aging, Germany, discussed how the protective effects of metformin on lifespan are abrogated in late-life interventions in C. elegans, which is likely caused by mitochondrial dysfunction associated with age. Increased mitochondrial content or ATP supplementation rescues the aging effect on metformin toxicity and restores its beneficial effects on healthy aging [23].

New findings have been made in dietary restriction mimetics as an easier therapeutic option. Sarah J. Mitchell, ETH Zürich, Switzerland, presented an alternative late-life intervention in 21 month old mice with the fumagillin derivative, ZGN1062, a methionine aminopeptidase 2 inhibitor, which showed positive effects on lifespan and healthspan, even more so than late-life calorie or methionine restriction. This suggested that the drug could be an alternative to calorie 
restriction (unpublished). Michael R. MacArthur, ETH Zurich, Switzerland, elaborated on their findings regarding ZGN1062. It rapidly decreases food-intake through activation of p53 signaling, leading to downstream induction of GDF15, and reduced food intake (unpublished). Guido Kroemer, Université de Paris, France, pointed out that caloric restriction mimetics enhances autophagy which improves longevity. Additionally, caloric restriction mimetics can be used in the treatment of diseases such as cancer; here the Kroemer group showed that the IGF1R inhibitor picropodophyllin enhance the efficacy of chemoimmunotherapy by stimulating immunosurveillance in mice [24, 25]. Valter Longo, USC Davis School of Gerontology, USA, discussed fasting mimicking diets in relation to vitamin $\mathrm{C}$ treatment of cancer cells. Vitamin C kills KRAS mutant cancer cells, but the effect is reduced by vitamin $\mathrm{C}$ activation of heme oxygenase 1 (HO-1) that induces ferritin which scavenges free $\mathrm{Fe}^{2+}$. This prevents the reaction of $\mathrm{Fe}^{2+}$ with $\mathrm{H}_{2} \mathrm{O}_{2}$ to produce hydroxy radicals. However, combined with a fasting mimicking diet the effects of vitamin $\mathrm{C}$ on HO-1 are inhibited, leading to increased levels of hydroxy radicals, increasing the efficiency of this treatment against cancer cells [26].

Pam Taub, University of California San Diego School of Medicine, USA, presented another alternative to DR. Time restricted eating is the alignment of eating patterns to the circadian rhythm, with a 8-10 hour daytime feeding window. This causes a metabolic shift from glucose to ketones leading to a low-grade state of ketosis which helps to build metabolic resilience, improves endothelial function and reduces inflammation. This metabolic resilience is beneficial against metabolic syndrome, and potentially also Covid-19 which shows similar hallmarks [27].

Further elucidating on the nutrient signaling pathways, Jens Brüning, Max Planck Institute for Metabolism Research, Germany, presented on the POMC and AgRP nutrient sensing neurons in the arcuate nucleus. The orexigenic AgRP is inhibited by insulin, and insulin binding to AgRP is necessary for normal insulin suppression of hepatic glucose production [28]. He further showed that insulin receptors on tanycytes are required for insulin transport to the CNS and actions in the arcuate nucleus (unpublished). Furthermore, Christian Riedel, Karolinska Institute, Sweden, showed that under reduced insulin/insulin-like growth factor signaling (ISS) in C. elegans, chromatin undergoes substantial accessibility changes. These promote activity of the transcription factor LIN-39 in neurons. It is proposed that LIN-39 is required for the formation or maintenance of neurons required for the longevity seen under reduced ISS (unpublished).

\section{Unlocking stem cell rejuvenation}

One characteristic of aging is stem cell exhaustion. Thus, understanding the properties and maintenance of stem cells may elucidate how to slow down the aging process. Maria Carolina Florian, Program for Regenerative Medicine, IDIBELL, Barcelona, Spain, discussed how aged hematopoietic stem cells (HSCs) localize in clusters away from the endosteum. Only functional aged HSCs are located at sinusoids, thus, the sinusoids are critical for their support, which is especially important to consider when treating with chemotherapy as it disrupts the sinusoids [29]. However, unlike other tissue, old HSCs are refractory to systemic rejuvenation interventions, as found by Emmannuella Passegué, Columbia University, USA. Old HSCs are not affected by exposure to young blood cells or known longevity interventions such as DR, and young HSCs are likewise not affected by old HSCs. This suggests that the focus should be on interventions to delay aging rather than rely on rejuvenation HSCs [30].

Alternatively, induction of pluripotent stem cells has been shown to rejuvenate tissue. Jean-Marc Lemaitre, Institute of Regenerative Medicine and Biotherapies of Montpellier, France, presented that even a short transient induction of the Yamanaka factors in a mouse model of premature aging early in life improved body composition and fitness throughout life as well as attenuated some of the aging related changes observed in the different tissues. The proposed mechanism is through epigenetic reprogramming in multiple organs [31]. On this topic, David A. Sinclair, Harvard Medical School, USA, showed that aging-driven epigenetic and gene expression changes in the central nervous system can be safely reversed to restore vision by inducible adeno-associated viruses expressing polycistronic Oct4, Sox 2 and Kif4, and that the effect was dependent on DNA demethylation [32]. Furthermore, rejuvenation can be obtained in vivo with partial reprogramming when Yamanaka factors are expressed for one week followed by a two-week recovery, as explained by Manuel Serrano, IRB Barcelona, Spain. However, the Serrano group is currently investigating how to rejuvenate independently of the Yamanaka factors. In addition, Serrano explained how repeated cycles of expression of the Yamanaka factors prevent the gradual loss of hippocampal neurogenesis associated to aging, and thereby it prevents the loss of memory performance of old mice. [33].

\section{Senolytics as an aging therapeutic}

Senescence is a known feature of aging, thus, the consequences of senescence as well as the use of 
senolytics as potential treatments are extensively studied. James Kirkland, Mayo Clinic, USA, described that transplanting of senescent cells in young mice caused spread of senescence to other cells and organs and led to increased frailty. The co-treatment of the senolytics dasatinib and quercetin broadly inhibited senescent cell anti-apoptotic pathways in mice, decreasing the number of naturally occurring senescence-associated secretory phenotype (SASP) presenting cells [34].

Furthering the discussion on SASP, tissue-specific knockout of the DNA repair endonuclease ERCC1-XPF revealed that the hematopoietic system, specifically, is a systemic driver of secondary senescence in a cell nonautonomous way through SASP, causing senescence to be found in non-immune cells in numerous organs, as presented by Laura Niedernhofer, University of Minnesota, USA. This makes senescent immune cells a good target for senolytics, such as fisetin, as well as potentially a good biomarker of tissue damage and multi-morbidities. She further showed that senescent cells in old mice cause an over-reaction to PAMPs, driving a cytokine storm that drives organ failure and death upon infection with a pathogen such as $\beta$-coronaviruses, an effect that can be ameliorated by treatment with the senolytic fisetin $[35,36]$. Eric Verdin from the Buck Institute, USA, showed how SASP promotes macrophage proliferation and macrophage senescence with age. This leads to an M1 switch which is key for macrophages involved in inflammaging. Macrophages should thus be considered as a potential therapeutic target [37]. The deleterious effects of SASP are seen across multiple tissues. Christina Manakanatas, University of Vienna, Austria, specifically explained how progerin expressing endothelial cells initiate a senescence signaling cascade mediate through the p53/p21 axis and miR34. This results in secretion of SASP and miR34 mediating paracrine signaling which could explain the cardiovascular diseases seen in HGPS (unpublished).

The potential of senotherapeutics is also emerging within the field of skin aging. The CSO of OneSkin Alessandra Zonari presented their latest findings regarding Peptide 14, identified through a cell-based screening. Treatment of aged skin with Peptide 14 caused a reduction in senescence and inflammatory markers and led to an increase in skin health with higher efficiency than rapamycin treatment [38]. Sibylle Jäger, L'Oreal Research and Innovation, presented a novel salicylic acid derivative C8-SA is known to promote longevity in C. elegans and prevent oxidative damage in humans by stimulating endogenous antioxidant defenses in an AMPK DAF-16 dependent manner (unpublished, L'Oreal).
From the point of senescent cells, Peter de Keizer, University Medical Center Utrecht, the Netherlands, stressed that senescence is heterogeneous and showed that specific subsets of senescent cells can be defined using imaging-mass cytometry (IMC), as well as realtime LMNB1-GFP reporter-based sorting and single cell RNA-seq. This, he argues, is crucial in the treatment of senescent cells. He showed their improved FOXO4-p53 DRI peptides have specificity for such a subset of senescent cells, called "scarred" cells - with striking effects in models for metastatic colon cancer and triple negative breast cancer [39].

\section{Looking at diverse models of aging}

As aging is multifaceted, using segmental models of aging can give us insight into different aspects of the many mechanisms of aging. A model system of aging can be an individual organ, other organisms, or a human disorder mimicking part of the aging process.

Brian Kennedy, National University of Singapore, Singapore, showed how Alzheimer's disease can be used as a model of neuronal aging. Increased lamin A expression is seen in aging and in Alzheimer's disease. ApoE KO in neuronal stem cells causes an increase in Lamin A and decrease in Lamin B. In astrocytes, this lead to accelerated senescence and increased inflammation which may affect neurons through an increase in inflammatory and senescence markers (unpublished).

Moving on to model organisms, Dario Valenzano, Max Planck Institute for Biology of Aging and Leibniz Institute on Aging, Germany, discussed the use of African turquoise killifish as a model of aging. Killifish show a diverse microbiome across different habitats and age, and their microbiome can be used as a readout of the health of the host. Treating killifish with the microbiome of a young fish has been shown to modulate lifespan [40, 41]. Vera Gorbunova, University of Rochester, USA, studies long lived mammals such as the bowhead whale and bats and has shown a strong link between longevity and genome maintenance, and specifically upregulation of double-stranded break (DSB) repair. While bowhead whales exhibit replicative senescence, they have attenuated SASP response and reduced inflammation. Additionally, their DSB repair efficiency is higher compared to humans (unpublished). Similarly, the little brown bat shows a high NHEJ efficiency compared to rats and a dampened inflammatory response $[42,43]$.

Daniela Bakula, University of Copenhagen, Denmark, presented how to use machine learning tools including aging clocks to identify diseases that show accelerated 
aging features. In this work they characterized a novel premature aging syndrome and investigated the underlying cellular function causing the observed accelerated aging pace (unpublished data).

\section{Tick, tock, aging clocks and biomarkers of aging}

A rapidly emerging method to study the aging process are aging clocks. Clocks can be trained on datasets extending from single cell -omics, movement data and blood parameters, to survey-based data and psychological factors. Anne Brunet, Stanford, USA, presented a single-cell RNA-seq based clock that can accurately predict chronological age across the neural stem cell niche and is very cell type specific. The clock is driven by cell-specific genes and it can be used to test rejuvenation interventions (unpublished). Furthermore, Alex Trapp, Harvard Medical School, USA, introduced the first single cell epigenetic clock that allows the aging process to be dissected in specific cell types. Additionally, an extension of the method called shallow sequencing decreased the sequencing cost compared to current deep epigenetic sequencing, while maintaining the accuracy [44]. Vadim Gladyshev, Harvard Medical School, USA, used multitissue epigenetic and aging clocks to show rejuvenation during early embryogenesis in mice and humans. He proposed a model wherein the germline ages during development and through adulthood but rejuvenates in the offspring after conception and reverts to the point of 'ground zero', which marks the beginning of aging $[45,46]$.

Georges Janssens, Amsterdam UMC, Netherlands, discussed the use of population data to create clocks. They created a movement clock using population data that predicts biological age. Additionally, the clock can be used to find factors such as diets or drugs associated with healthier aging (unpublished). Alex Zhavoronkov, Insilico Medicine, Hong Kong, presented the many aging clocks developed by Deep Longevity as well as their newest clock, MindAge, which is a psychological aging clock. MindAge is trained on survey-based data with the purpose of being used in clinics, by insurance companies, and by employers [47]. Kai-Fu Lee, CEO Sinovation Ventures, Beijing, China also advocated the use of aging clocks in the clinics, however, he emphasized the need for more specific clocks trained on better data-sets and combining multiple clocks. Many clocks such as GlycanAge, PhotoAge and BloodAge are used in the clinic in order to predict the biological age of the patient, as explained by Dina Radenkovic, Hooke London by Health and Longevity Optimisation, UK. Despite a believed high predictive value, guidelines should be created on how to apply the clocks to a clinical setting [48].
TruDiagnostic has developed a clock that predicts the pace of aging rather than true biological age as shown by Ryan Smith. DunedinPACE is a blood DNA methylation clock showing pace of aging targeted for individual persons [49].

Aging clocks are a key factor in order to assess human health and identifying promising interventions. However, Brian Chen from FOXO Technologies, USA stressed the importance of validation and systematic characterization of described clocks - like all other biomarkers - will be essential to drive the aging industry forward [50].

The evaluation of the efficacy of aging interventions requires a combination of different molecular biomarkers. A specific population of interest in the study of biomarkers are centenarians, as studied by Yousin Suh, Columbia University, USA. She has examined centenarian gene variants in conserved pathways of aging to find alleles that promote longevity such as the longevity-associated variant of SMAD3gene, which causes decreased expression of SMAD3 thereby preventing the effects on lifespan and senescence (unpublished).

An additional approach to discover biomarkers was presented by Anastasia Georgievskaya, Haut.AI, Estonia. Using AI to discover visual, facial biomarkers that are gender and age specific can be obtained from a selfie. These biomarkers can potentially be used to measure the effect of longevity interventions in a clinical setting [51]. Rudi Westendorp, University of Copenhagen, Denmark, proposed that our current biomarkers change with age and have only little predictive value for clinical prediction. His group has developed a recurrent neural network to predict frailty and subsequent need for preventative services in old persons which showed better predictive value compared to established methods [52]. An alternative approach, using dynamic indicators of the response to stressors seem to provide more information on resilience of organisms (unpublished).

\section{Rethinking aging research: from preclinical to clinical}

Effective aging therapeutics for humans are dependent on the pipeline from drug discovery to testing in model organisms and through to human clinical trials. By advancing on how we approach drug discovery and the transition from preclinical to clinical trials, we can expect to see cheaper, faster, and more effective therapeutics emerge.

The use of AI has led to broader and less biased approaches for the discovery of potential therapeutics. 
Ivan Ozerov, Insilico Medicine, Hong Kong, presented PandOmics as a platform that uses an AI basedapproach to discover new therapeutic targets for different diseases and disease subtypes. This can ease and speed up the discovery of potential drug candidates [53]. Morten Scheibye-Knudsen, University of Copenhagen, Denmark further highlighted the importance of AI-based approaches in finding lifespanimproving drugs. Using Danish population-based prescription data, the Scheibye-Knudsen lab has identified drugs associated with longer lifespan. Additionally, by using in silico-based high-throughput screening, potential therapeutics that stimulate ageassociated DNA repair pathways have been characterized (unpublished). Alice Ruixue Ai, University of Oslo and Akershus University Hospital, Norway, showed an AI-based approach to identify potent mitophagy inducers. The two lead compounds diminished Tau pathology and improved the memory of an Alzheimer's disease mouse model, thus, highlighting the potential of use of AI in a preclinical setting (unpublished).

Optimizing and upscaling methods of preclinical testing will allow faster and cheaper drug discovery. Gordian Biotechnology, USA, created a setup that simultaneously tests the in vivo efficacy of multiple drugs prior to clinical testing using pooled in vivo screening, as presented by Martin Borch Jensen. He highlights this as a way to increase the likelihood of a drug succeeding through clinical trials [54]. Calico has developed a semi-automated platform for measuring healthspan in mice and it can be used to detect several aging phenotypes, easing the labor-intensive and time consuming testing of longevity drugs in mice, as shown by Adam Freund [55].

Matt Kaeberlein, The University of Washington, USA, presented their new WormBot, a set it and forget it method of large-scale intervention testing in C. elegans. $\mathrm{He}$ stressed the importance of broad and unbiased screening of intervention beyond known pathways and in different combinations [56]. Additionally, a high throughput, fully automated C. elegans organism-onchip method that can be used for lead optimization, early toxicology and drug discovery was described by David Liaskos, The École Polytechnique Fédérale de Lausanne (EPFL), Switzerland. Development, reproduction, motility and survival information can be extracted resulting in faster preclinical testing [57]. Michael Petr Tracked.Bio, presented their use of AIbased software to track motion data of drosophila and mice, which can be used to study movement as features of healthspan as well as survival data [58].

The transition from preclinical to clinical studies remains a challenging and protracted process. Andrea
Maier, National University of Singapore, Singapore, stressed the need for an unbiased standardized characterization and reporting of research outcome in the form of a minimum required dataset and trial networks to combat the poor transition currently seen. Additionally, she suggested drug repurposing as a relatively cheap and faster way for new age-related treatments $[59,60]$. The great benefit of drug repurposing was also highlighted by Nir Barzilai, Albert Einstein College of Medicine, USA, who studies the repurposing of FDA approved drugs such as metformin and canagliflozin [61]. As aging is not currently recognized as a disease by the FDA, drug repurposing is an invaluable tool [62]. Anne-Ulrike Trendelenburg, Novartis Institutes for Biomedical Research, USA, presented a roadmap on how to conduct preclinical testing of geroprotectors for easy clinical translation. She highlighted biomarkers for frailty and targeting multi morbidity as crucial points to consider during preclinical testing of gerotherapeutics [63, 64].

Furthering the discussion on new common standards of studies, Luigi Ferrucci, NIA-NIH, USA, presented the value of using longitudinal perspectives in population studies. Longitudinal studies are more precise for measuring biomarkers and factors of aging, and looking specifically at longitudinal changes and trajectories in aging allows the study of functions of aging across lifespan [65].

\section{Longevity industry landscape}

This year at the conference, multiple companies presented their way of tackling drug discovery. James Peyer, CEO of Cambrian biopharma, a Distributed Development Company, discussed their strategy to use established regulatory frameworks in order to develop new healthspan extending medicines. They have established a pipeline of new drugs that target causes of aging and age-related diseases and have candidates for clinical testing [66]. Furthermore, the CEO of BioAge, Kristen Fortney presented their pipeline that starts with analyzing human longitudinal data and ends with clinical testing of a validated compound to discover treatments that can extend healthy lifespan by targeting the molecular causes of aging. This has currently led to three compounds in clinical trials [67].

Moreover, new ways of funding are emerging such as VitaDAO, a new decentralized collective funding of early-stage longevity research as presented by cofounder Tylor Golato. Their goal is to extend human lifespan by researching, financing, and commercializing longevity therapeutics in an open and democratic manner [68]. Also, among venture capitalists there is an increasing awareness of the aging field. Kris Verburgh 
from the Longevity Vision Fund presented their work in investing in technologies to extend lifespan and improve healthspan [69].

\section{CONCLUSION}

Aging is a multifaceted process, making research complex and diverse in topics. This year's conference has elucidated the latest findings of aging mechanisms using known and novel models of aging and the modulation of these and understanding how to slow aging by stem cell rejuvenation or senotherapeutics. Finally, the latest advances in AI were presented as well as new ideas on how to facilitate the transition from preclinical to clinical studies. This effort is shared by an emerging industry involved in every step of the research journey making the prospect of healthy aging very real. The future is bright.

\section{CONFLICTS OF INTEREST}

S.J.M. and M.R.M. declare no conflicts of interest. D.A.S. is a founder, board member and equity owner of Life Biosciences. His other activities are disclosed at https://sinclair.hms.harvard.edu/david-sinclairs-affiliations. B.H.C. is an employee of FOXO Technologies Inc.

\section{FUNDING}

S.B.J. was supported the European Research Council (ERC) under the European Union's Horizon 2020 research and innovation program (grant agreement 863911 - PHYRIST). M.K. was support by NIH grant P30AG013280. S.J.M. and M.R.M. were supported through a research agreement with Zafgen Inc (Boston MA). R.W. Dr is supported by the Novo Nordisk Foundation Challenge Programme: Harnessing the Power of Big Data to Address the Societal Challenge of Aging [NNF17OC0027812]. V.N.G is supported by NIH grants xx. F.C. is supported by the Wellcome Trust/Royal Society $\quad(102532 / \mathrm{Z} / 12 / \mathrm{Z}$ and 102531/Z/13/A) and MRC (MC-A654-5QC80). L.P. and Y.X.L. are supported by the European Research Council (ERC) under the European Union's Horizon 2020 research and innovation programme no. 741989 and the Max-Planck-Gesellschaft. Y.X.L. is supported by an EMBO Long-Term Fellowship (ALTF 4192014). C.G.R. is supported by grants from the Swedish Research Council (VR) (2015-03740, 2017-06088, and 2019-04868), the Swedish Cancer Society (Cancerfonden) $(201034 \mathrm{Pj})$, the Novo Nordisk Foundation (NNF21OC0070427), and by an ICMC project grant. D.B. is supported by the Lundbeck foundation (\#R303-2018-3159). M.S.K. is supported by the Novo Nordisk Foundation Challenge Programme (\#NNF17OC0027812), the Nordea Foundation (\#02-
2017-1749), the Neye Foundation, the Lundbeck Foundation (\#R324-2019-1492), the Ministry of Higher Education and Science (\#0238-00003B), VitaDAO and Insilico Medicine. We would also like to offer our sincere thanks to our amazing sponsors and all the volunteers that made this meeting possible.

\section{REFERENCES}

1. López-Otín C, Blasco MA, Partridge L, Serrano M, Kroemer G. The hallmarks of aging. Cell. 2013; 153:1194-217.

https://doi.org/10.1016/i.cell.2013.05.039

PMID:23746838

2. Vijg J, Dong X. Pathogenic Mechanisms of Somatic Mutation and Genome Mosaicism in Aging. Cell. 2020; 182:12-23.

https://doi.org/10.1016/i.cell.2020.06.024 PMID:32649873

3. Wang S, Meyer DH, Schumacher B. H3K4me2 regulates the recovery of protein biosynthesis and homeostasis following DNA damage. Nat Struct Mol Biol. 2020; 27:1165-77. https://doi.org/10.1038/s41594-020-00513-1 PMID:33046905

4. Martinez-Miguel VE, Lujan C, Espie-Caullet T, MartinezMartinez D, Moore S, Backes C, Gonzalez S, Galimov ER, Brown AEX, Halic M, Tomita K, Rallis C, von der Haar $T$, et al. Increased fidelity of protein synthesis extends lifespan. Cell Metab. 2021; 33:2288-300.e12.

https://doi.org/10.1016/j.cmet.2021.08.017 PMID:34525330

5. Buchwalter A, Hetzer MW. Nucleolar expansion and elevated protein translation in premature aging. Nat Commun. 2017; 8:328.

https://doi.org/10.1038/s41467-017-00322-z PMID:28855503

6. Lu YX, Regan JC, Eßer J, Drews LF, Weinseis T, Stinn J, Hahn O, Miller RA, Grönke S, Partridge L. A TORC1histone axis regulates chromatin organisation and non-canonical induction of autophagy to ameliorate ageing. Elife. 2021; 10:e62233.

https://doi.org/10.7554/eLife.62233

PMID:33988501

7. Luongo TS, Eller JM, Lu MJ, Niere M, Raith F, Perry C, Bornstein MR, Oliphint P, Wang L, McReynolds MR, Migaud ME, Rabinowitz JD, Johnson FB, et al. SLC25A51 is a mammalian mitochondrial $\mathrm{NAD}^{+}$ transporter. Nature. 2020; 588:174-9. https://doi.org/10.1038/s41586-020-2741-7 PMID:32906142

8. Scheibye-Knudsen M, Mitchell SJ, Fang EF, Iyama T, Ward T, Wang J, Dunn CA, Singh N, Veith S, Hasan- 
Olive MM, Mangerich A, Wilson MA, Mattson MP, et al. A high-fat diet and $N A D(+)$ activate Sirt1 to rescue premature aging in cockayne syndrome. Cell Metab. 2014; 20:840-55.

https://doi.org/10.1016/j.cmet.2014.10.005

PMID:25440059

9. Yang B, Dan $X$, Hou $Y$, Lee JH, Wechter $N$, Krishnamurthy $S$, Kimura $R$, Babbar $M$, Demarest $T$, McDevitt $R$, Zhang $S$, Zhang $Y$, Mattson MP, et al. $\mathrm{NAD}^{+}$supplementation prevents STING-induced senescence in ataxia telangiectasia by improving mitophagy. Aging Cell. 2021; 20:e13329.

https://doi.org/10.1111/acel.13329

PMID:33734555

10. Zapata-Pérez R, Tammaro A, Schomakers BV, Scantlebery AML, Denis S, Elfrink HL, GiroudGerbetant J, Cantó C, López-Leonardo C, McIntyre RL, van Weeghel M, Sánchez-Ferrer Á, Houtkooper RH. Reduced nicotinamide mononucleotide is a new and potent $\mathrm{NAD}^{+}$precursor in mammalian cells and mice. FASEB J. 2021; 35:e21456.

https://doi.org/10.1096/fj.202001826R PMID:33724555

11. Yuan J, Chang SY, Yin SG, Liu ZY, Cheng X, Liu XJ, Jiang $Q$, Gao G, Lin DY, Kang XL, Ye SW, Chen Z, Yin JA, et al. Two conserved epigenetic regulators prevent healthy ageing. Nature. 2020; 579:118-22.

https://doi.org/10.1038/s41586-020-2037-y PMID: 32103178

12. Angeli S, Foulger A, Chamoli M, Peiris TH, Gerencser A, Shahmirzadi AA, Andersen J, Lithgow G. The mitochondrial permeability transition pore activates the mitochondrial unfolded protein response and promotes aging. Elife. 2021; 10:e63453.

https://doi.org/10.7554/eLife.63453 PMID: $\underline{34467850}$

13. Statzer C, Jongsma E, Liu SX, Dakhovnik A, Wandrey F, Mozharovskyi P, Zülli F, Ewald CY. Youthful and agerelated matreotypes predict drugs promoting longevity. Aging Cell. 2021; 20:e13441.

https://doi.org/10.1111/acel.13441

PMID:34346557

14. Shaposhnikov MV, Zemskaya NV, Koval LA, Schegoleva EV, Yakovleva DV, Ulyasheva NS, Gorbunova AA, Minnikhanova NR, Moskalev AA. Geroprotective potential of genetic and pharmacological interventions to endogenous hydrogen sulfide synthesis in Drosophila melanogaster. Biogerontology. 2021; 22:197-214. https://doi.org/10.1007/s10522-021-09911-4 PMID:33544267

15. Obri A, Khrimian L, Karsenty G, Oury F. Osteocalcin in the brain: from embryonic development to age- related decline in cognition. Nat Rev Endocrinol. 2018; 14:174-82.

https://doi.org/10.1038/nrendo.2017.181

PMID:29376523

16. Annibal A, Tharyan RG, Schonewolff MF, Tam H, Latza C, Auler MMK, Grönke S, Partridge L, Antebi A. Regulation of the one carbon folate cycle as a shared metabolic signature of longevity. Nat Commun. 2021; 12:3486.

https://doi.org/10.1038/s41467-021-23856-9 PMID: $\underline{34108489}$

17. McAleavy $M$, Zhang $Q$, Xu J, Pan L, Wakai M, Ehmann PJ, Wipperman MF, Shavlakadze T, Hamon SC, Boyapati A, Morton LG, Kyratsous CA, Glass DJ. Activin A correlates with the worst outcomes in COVID-19 patients, and can be induced by cytokines via the IKK/NF-kappa B pathway. bioRxiv. 2021. [Epub ahead of print].

https://doi.org/10.1101/2021.02.04.429815

18. Rattan SI. Hormesis in aging. Ageing Res Rev. 2008; 7:63-78.

https://doi.org/10.1016/i.arr.2007.03.002

PMID:17964227

19. Karlsen A, Soendenbroe C, Malmgaard-Clausen NM, Wagener F, Moeller CE, Senhaji Z, Damberg K, Andersen JL, Schjerling $\mathrm{P}$, Kjaer $\mathrm{M}$, Mackey AL. Preserved capacity for satellite cell proliferation, regeneration, and hypertrophy in the skeletal muscle of healthy elderly men. FASEB J. 2020; 34:6418-36.

https://doi.org/10.1096/fj.202000196R

PMID:32167202

20. Vind AC, Snieckute G, Blasius M, Tiedje C, Krogh N, Bekker-Jensen DB, Andersen KL, Nordgaard C, Tollenaere MAX, Lund AH, Olsen JV, Nielsen $H$, Bekker-Jensen S. ZAKa Recognizes Stalled Ribosomes through Partially Redundant Sensor Domains. Mol Cell. 2020; 78:700-13.e7.

https://doi.org/10.1016/j.molcel.2020.03.021 PMID:32289254

21. Birkisdóttir MB, Jaarsma D, Brandt RMC, Barnhoorn $S$, van Vliet $\mathrm{N}$, Imholz S, van Oostrom $\mathrm{CT}$, Nagarajah $\mathrm{B}$, Portilla Fernández E, Roks AJM, Elgersma Y, van Steeg $H$, Ferreira JA, et al. Unlike dietary restriction, rapamycin fails to extend lifespan and reduce transcription stress in progeroid DNA repair-deficient mice. Aging Cell. 2021; 20:e13302.

https://doi.org/10.1111/acel.13302

PMID: $\underline{3484480}$

22. Rodgers JT, King KY, Brett JO, Cromie MJ, Charville GW, Maguire KK, Brunson C, Mastey N, Liu L, Tsai CR, Goodell MA, Rando TA. mTORC1 controls the adaptive transition of quiescent stem cells from $\mathrm{GO}$ to G(Alert). Nature. 2014; 510:393-6. 
https://doi.org/10.1038/nature13255

PMID:24870234

23. Espada L, Dakhovnik A, Chaudhari P, Martirosyan A, Miek L, Poliezhaieva T, Schaub Y, Nair A, Döring N, Rahnis N, Werz O, Koeberle A, Kirkpatrick J, et al. Loss of metabolic plasticity underlies metformin toxicity in aged Caenorhabditis elegans. Nat Metab. 2020; 2:1316-31.

https://doi.org/10.1038/s42255-020-00307-1

PMID:33139960

24. Wu Q, Tian AL, Durand $S$, Aprahamian F, Nirmalathasan N, Xie W, Liu P, Zhao L, Zhang S, Pan $\mathrm{H}$, Carmona-Gutierrez D, Madeo F, Tu Y, et al. Isobacachalcone induces autophagy and improves the outcome of immunogenic chemotherapy. Cell Death Dis. 2020; 11:1015.

https://doi.org/10.1038/s41419-020-03226-x

PMID:33243998

25. Wu Q, Tian AL, Li B, Leduc $M$, Forveille $S$, Hamley $P$, Galloway W, Xie W, Liu P, Zhao L, Zhang S, Hui P, Madeo $F$, et al. IGF1 receptor inhibition amplifies the effects of cancer drugs by autophagy and immunedependent mechanisms. J Immunother Cancer. 2021; 9:e002722.

https://doi.org/10.1136/iitc-2021-002722 PMID:34127545

26. Di Tano M, Raucci F, Vernieri C, Caffa I, Buono R, Fanti M, Brandhorst S, Curigliano G, Nencioni A, de Braud $F$, Longo VD. Synergistic effect of fasting-mimicking diet and vitamin $\mathrm{C}$ against KRAS mutated cancers. Nat Commun. 2020; 11:2332.

https://doi.org/10.1038/s41467-020-16243-3 PMID: $\underline{32393788}$

27. Świątkiewicz I, Woźniak A, Taub PR. Time-Restricted Eating and Metabolic Syndrome: Current Status and Future Perspectives. Nutrients. 2021; 13:221.

https://doi.org/10.3390/nu13010221

PMID:33466692

28. Steculorum SM, Ruud J, Karakasilioti I, Backes H, Engström Ruud L, Timper K, Hess ME, Tsaousidou E, Mauer J, Vogt MC, Paeger L, Bremser S, Klein AC, et al. AgRP Neurons Control Systemic Insulin Sensitivity via Myostatin Expression in Brown Adipose Tissue. Cell. 2016; 165:125-38.

https://doi.org/10.1016/i.cell.2016.02.044

PMID:27015310

29. Saçma M, Pospiech J, Bogeska R, de Back W, Mallm JP, Sakk V, Soller K, Marka G, Vollmer A, Karns R, CabezasWallscheid N, Trumpp A, Méndez-Ferrer S, et al. Haematopoietic stem cells in perisinusoidal niches are protected from ageing. Nat Cell Biol. 2019; 21:1309-20. https://doi.org/10.1038/s41556-019-0418-y PMID: $\underline{31685996}$
30. Ho TT, Dellorusso PV, Verovskaya EV, Bakker ST, Flach J, Smith LK, Ventura PB, Lansinger OM, Hérault $A$, Zhang SY, Kang YA, Mitchell CA, Villeda SA, Passegué E. Aged hematopoietic stem cells are refractory to bloodborne systemic rejuvenation interventions. J Exp Med. 2021; 218:e20210223.

https://doi.org/10.1084/jem.20210223

PMID:34032859

31. Alle $\mathrm{Q}$, Le Borgne $\mathrm{E}$, Bensadoun $\mathrm{P}$, Lemey $\mathrm{C}$, Béchir $\mathrm{N}$, Gabanou M, Estermann F, Bertrand-Gaday C, Pessemesse L, Toupet K, Vialaret J, Hirtz C, Noël D, et al. A single short reprogramming early in life improves fitness and increases lifespan in old age. bioRxiv. 2021. [Epub ahead of print]. https://doi.org/10.1101/2021.05.13.443979

32. Lu Y, Brommer B, Tian X, Krishnan A, Meer M, Wang C, Vera DL, Zeng Q, Yu D, Bonkowski MS, Yang JH, Zhou S, Hoffmann EM, et al. Reprogramming to recover youthful epigenetic information and restore vision. Nature. 2020; 588:124-9.

https://doi.org/10.1038/s41586-020-2975-4

PMID:33268865

33. Rodríguez-Matellán A, Alcazar N, Hernández $F$, Serrano $M$, Ávila J. In Vivo Reprogramming Ameliorates Aging Features in Dentate Gyrus Cells and Improves Memory in Mice. Stem Cell Reports. 2020; 15:1056-66.

https://doi.org/10.1016/i.stemcr.2020.09.010

PMID:33096049

34. Xu M, Pirtskhalava T, Farr JN, Weigand BM, Palmer AK, Weivoda MM, Inman CL, Ogrodnik MB, Hachfeld CM, Fraser DG, Onken JL, Johnson KO, Verzosa GC, et al. Senolytics improve physical function and increase lifespan in old age. Nat Med. 2018; 24:1246-56.

https://doi.org/10.1038/s41591-018-0092-9 PMID:29988130

35. Yousefzadeh MJ, Flores RR, Zhu Y, Schmiechen ZC, Brooks RW, Trussoni CE, Cui Y, Angelini L, Lee KA, McGowan SJ, Burrack AL, Wang D, Dong $Q$, et al. An aged immune system drives senescence and ageing of solid organs. Nature. 2021; 594:100-5.

https://doi.org/10.1038/s41586-021-03547-7 PMID:33981041

36. Camell CD, Yousefzadeh MJ, Zhu Y, Prata LGP, Huggins MA, Pierson M, Zhang L, O'Kelly RD, Pirtskhalava T, Xun P, Ejima K, Xue A, Tripathi U, et al. Senolytics reduce coronavirus-related mortality in old mice. Science. 2021; 373:eabe4832. https://doi.org/10.1126/science.abe4832 PMID:34103349

37. Covarrubias AJ, Kale A, Perrone R, Lopez-Dominguez JA, Pisco AO, Kasler HG, Schmidt MS, Heckenbach I, 
Kwok R, Wiley CD, Wong HS, Gibbs E, lyer SS, et al. Senescent cells promote tissue $\mathrm{NAD}^{+}$decline during ageing via the activation of $\mathrm{CD} 38^{+}$macrophages. Nat Metab. 2020; 2:1265-83.

https://doi.org/10.1038/s42255-020-00305-3 PMID:33199924

38. Zonari A, Brace LE, Al-Katib KZ, Porto WF, Foyt D, Guiang $M$, Cruz EAO, Marshall B, Salgueiro WG, Inan MD, Rahman M, Anupom T, Vanapalli S, et al. Senotherapeutic peptide reduces skin biological age and improves skin health markers. bioRxiv. 2020. [Epub ahead of print]. https://doi.org/10.1101/2020.10.30.362822

39. Baar MP, Brandt RMC, Putavet DA, Klein JDD, Derks KWJ, Bourgeois BRM, Stryeck S, Rijksen $Y$, van Willigenburg H, Feijtel DA, van der Pluijm I, Essers J, van Cappellen WA, et al. Targeted Apoptosis of Senescent Cells Restores Tissue Homeostasis in Response to Chemotoxicity and Aging. Cell. 2017; 169:132-47.e16.

https://doi.org/10.1016/i.cell.2017.02.031

PMID:28340339

40. Valenzano DR, Benayoun BA, Singh PP, Zhang E, Etter PD, Hu CK, Clément-Ziza M, Willemsen D, Cui R, Harel I, Machado BE, Yee MC, Sharp SC, et al. The African Turquoise Killifish Genome Provides Insights into Evolution and Genetic Architecture of Lifespan. Cell. 2015; 163:1539-54.

https://doi.org/10.1016/j.cell.2015.11.008 PMID:26638078

41. Smith P, Willemsen D, Popkes M, Metge F, Gandiwa $E$, Reichard M, Valenzano DR. Regulation of life span by the gut microbiota in the short-lived African turquoise killifish. Elife. 2017; 6:e27014.

https://doi.org/10.7554/eLife.27014

PMID:28826469

42. Gorbunova V, Seluanov A, Kennedy BK. The World Goes Bats: Living Longer and Tolerating Viruses. Cell Metab. 2020; 32:31-43.

https://doi.org/10.1016/j.cmet.2020.06.013 PMID:32640245

43. Zhao Y, Seluanov A, Gorbunova V. Revelations About Aging and Disease from Unconventional Vertebrate Model Organisms. Annu Rev Genet. 2021; 55:135-59. https://doi.org/10.1146/annurev-genet-071719$\underline{021009}$ PMID:34416119

44. Trapp A, Kerepesi C, Gladyshev VN. Profiling epigenetic age in single cells. bioRxiv. 2021. [Epub ahead of print]. https://doi.org/10.1101/2021.03.13.435247

45. Gladyshev VN. The Ground Zero of Organismal Life and Aging. Trends Mol Med. 2021; 27:11-9. https://doi.org/10.1016/j.molmed.2020.08.012 PMID:32980264

46. Kerepesi C, Zhang B, Lee SG, Trapp A, Gladyshev VN. Epigenetic clocks reveal a rejuvenation event during embryogenesis followed by aging. Sci Adv. 2021; 7:eabg6082.

https://doi.org/10.1126/sciadv.abg6082

PMID: $\underline{34172448}$

47. Zhavoronkov A, Kochetov K, Diamandis P, Mitina M. PsychoAge and SubjAge: development of deep markers of psychological and subjective age using artificial intelligence. Aging (Albany NY). 2020; 12:23548-77.

https://doi.org/10.18632/aging.202344

PMID:33303702

48. Krištić J, Vučković F, Menni C, Klarić L, Keser T, Beceheli I, Pučić-Baković $M$, Novokmet $M$, Mangino M, Thaqi K, Rudan P, Novokmet N, Sarac J, et al. Glycans are a novel biomarker of chronological and biological ages. J Gerontol A Biol Sci Med Sci. 2014; 69:779-89.

https://doi.org/10.1093/gerona/glt190 PMID:24325898

49. TruDiagnostics. https://trudiagnostic.com/.

50. Quach A, Levine ME, Tanaka T, Lu AT, Chen BH, Ferrucci L, Ritz B, Bandinelli S, Neuhouser ML, Beasley JM, Snetselaar L, Wallace RB, Tsao PS, et al. Epigenetic clock analysis of diet, exercise, education, and lifestyle factors. Aging (Albany NY). 2017; 9:419-46.

https://doi.org/10.18632/aging.101168

PMID:28198702

51. Bobrov E, Georgievskaya A, Kiselev K, Sevastopolsky A, Zhavoronkov A, Gurov S, Rudakov K, Del Pilar Bonilla Tobar $M$, Jaspers $S$, Clemann $S$. PhotoAgeClock: deep learning algorithms for development of non-invasive visual biomarkers of aging. Aging (Albany NY). 2018; 10:3249-59.

https://doi.org/10.18632/aging.101629

PMID:30414596

52. Wright MN, Kusumastuti S, Mortensen L, Westendorp RGJ, Gerds T. Personalised need of care in an ageing society: The making of a prediction tool based on register data. J R Stat Soc Ser A Stat Soc. 2021; 184:1199-1219. https://doi.org/10.1111/rssa.12644

53. Ozerov IV, Lezhnina KV, Izumchenko E, Artemov AV, Medintsev S, Vanhaelen Q, Aliper A, Vijg J, Osipov AN, Labat I, West MD, Buzdin A, Cantor CR, et al. In silico Pathway Activation Network Decomposition Analysis (iPANDA) as a method for biomarker development. Nat Commun. 2016; 7:13427. https://doi.org/10.1038/ncomms13427 
PMID:27848968

54. Jensen MB, Marblestone A. In vivo Pooled Screening: A Scalable Tool to Study the Complexity of Aging and Age-Related Disease. Front Aging. 2021; 2:714926. https://doi.org/10.3389/fragi.2021.714926

55. Chen Z, Raj A, Prateek GV, Francesco AD, Liu J, Keyes $B E$, Kolumam G, Jojic V, Freund A. Automated, highdimensional evaluation of physiological aging and resilience in outbred mice. bioRxiv. 2021. [Epub ahead of print].

https://doi.org/10.1101/2021.08.02.454830

56. Pitt JN, Strait NL, Vayndorf EM, Blue BW, Tran $\mathrm{CH}$, Davis BEM, Huang K, Johnson BJ, Lim KM, Liu $S$, Nikjoo A, Vaid A, Wu JZ, Kaeberlein M. WormBot, an open-source robotics platform for survival and behavior analysis in C. elegans. Geroscience. 2019; 41:961-73.

https://doi.org/10.1007/s11357-019-00124-9

PMID:31728898

57. Nagi Bioscience. https://nagibio.ch/.

58. Tracked.bio. http://www.tracked.bio/.

59. Heard DS, Tuttle CSL, Lautenschlager NT, Maier AB. Repurposing Proteostasis-Modifying Drugs to Prevent or Treat Age-Related Dementia: A Systematic Review. Front Physiol. 2018; 9:1520.

https://doi.org/10.3389/fphys.2018.01520

PMID:30425653

60. Kioussis B, Tuttle CSL, Heard DS, Kennedy BK, Lautenschlager NT, Maier AB. Targeting impaired nutrient sensing with repurposed therapeutics to prevent or treat age-related cognitive decline and dementia: A systematic review. Ageing Res Rev. 2021; 67:101302.

https://doi.org/10.1016/j.arr.2021.101302 PMID: 33609776

61. Kulkarni AS, Gubbi S, Barzilai N. Benefits of Metformin in Attenuating the Hallmarks of Aging. Cell Metab. 2020; 32:15-30. https://doi.org/10.1016/i.cmet.2020.04.001 PMID: $\underline{32333835}$
62. DeVito LM, Barzilai N, Cuervo AM, Niedernhofer LJ, Milman S, Levine $M$, Promislow D, Ferrucci $L$, Kuchel GA, Mannick J, Justice J, Gonzales MM, Kirkland JL, et al. Extending human healthspan and longevity: a symposium report. Ann N Y Acad Sci. 2022; 1507:7083.

https://doi.org/10.1111/nyas.14681

PMID: 34498278

63. von Zglinicki T, Varela Nieto I, Brites D, Karagianni N, Ortolano S, Georgopoulos S, Cardoso AL, Novella S, Lepperdinger G, Trendelenburg AU, van Os R. Frailty in mouse ageing: A conceptual approach. Mech Ageing Dev. 2016; 160:34-40.

https://doi.org/10.1016/i.mad.2016.07.004

PMID:27443148

64. Cardoso AL, Fernandes A, Aguilar-Pimentel JA, de Angelis MH, Guedes JR, Brito MA, Ortolano S, Pani G, Athanasopoulou S, Gonos ES, Schosserer M, Grillari J, Peterson P, et al. Towards frailty biomarkers: Candidates from genes and pathways regulated in aging and age-related diseases. Ageing Res Rev. 2018; 47:214-77.

https://doi.org/10.1016/i.arr.2018.07.004 PMID: $\underline{30071357}$

65. Kuo PL, Schrack JA, Shardell MD, Levine M, Moore AZ, An Y, Elango P, Karikkineth A, Tanaka T, de Cabo R, Zukley LM, AlGhatrif $M$, Chia CW, et al. A roadmap to build a phenotypic metric of ageing: insights from the Baltimore Longitudinal Study of Aging. J Intern Med. 2020; 287:373-94. https://doi.org/10.1111/joim.13024 PMID: $\underline{32107805}$

66. Cambrian. https://www.cambrianbio.com/.

67. BioAge. https://bioagelabs.com/.

68. VitaDAO. https://www.vitadao.com/.

69. Longevity Vision Fund. https://Ivf.vc/. 